\title{
A Discontinuous Galerkin Formulation for Solution of Parabolic Equations on Nonconforming Meshes
}

\author{
Deepak V. Kulkarni, Dimitrios V. Rovas, and Daniel A. Tortorelli * \\ 1206 West Green St., Mech. \& Industr. Engrg. Dept., University of Illinois, Urbana, IL \\ 61801, USA. \{dkulkarn, rovas, dtortore\}euiuc.edu
}

Summary. Non-conforming meshes are frequently employed in multi-component simulations and adaptive refinement. In this work we develop a discontinuous Galerkin framework capable of accommodating non-conforming meshes and apply our approach to analyzing the transient heat conduction problem.

\section{Introduction}

Non-conforming meshes are frequently employed for adaptive solution or simulation of multi-component systems. Even though non-conforming meshes are easy to generate, they require the satisfaction of jump conditions across the non-conforming mesh interface. Several techniques have been developed to enforce these conditions such as mixed methods (Arbogast [1997]), local constraint equation methods (Guo and Babuška [1986], Demkowicz et al. [1989]) and mortar methods (Bernadi et al. [1989], Wohlmuth [2002]).

In this work we present a Discontinuous Galerkin (DG) framework for accommodating non-conforming meshes. The DG method naturally accommodates jump conditions and has been employed to solve hyperbolic, parabolic and elliptic problems (Cockburn et al. [2000]). For a historical review of DG methods and their applications to elliptic problems refer to Arnold et al. [2002]. Recently, DG schemes have been applied to enforce jump conditions across non-conforming mesh interfaces such as those encountered in adaptive refinement (Becker and Hansbo [1999], Carmo and Duarte [2000], Perugia and Schötzau [2001]). Here, we extend the formulation of Becker and Hansbo [1999] to parabolic problems. A benefit of the DG scheme is that it does not introduce constraint equations and their resulting Lagrange multiplier fields, as done in mixed and mortar methods. However, the standard DG formulation leads to a large system of equations due to the presence of "duplicate" nodes.

\footnotetext{
* The first and third author would like to acknowledge the support provided by NSF under grant no. DMR 01-21695
} 
In sections (2) and (3) we describe our discontinuous Galerkin (DG) formulation and provide an a priori analysis. Section (4) presents numerical examples using our formulation. Finally, in section (5) we draw conclusions and suggest future work.

\section{Problem Definition}

We consider the following linear heat conduction problem as our representative example of a parabolic equation:

$$
\begin{aligned}
\dot{u}-\Delta u=f & \text { in } \Omega \times I \\
u=0 & \text { on } \partial \Omega_{D} \times I \\
-\nabla u \cdot \mathbf{n}=0 & \text { on } \partial \Omega_{N} \times I \\
u(\cdot, 0)=\tilde{u} & \text { in } \Omega
\end{aligned}
$$

where $u(x, t)$ is the scalar temperature field to be computed over the time interval $I=(0, T) ; \partial \Omega$ defines the boundary of the region $\Omega$ which is divided into two complimentary regions, $\partial \Omega_{D}$, on which homogeneous Dirichlet boundary conditions are specified, and $\partial \Omega_{N}$, on which homogeneous Neumann boundary conditions are specified; and $\tilde{u}$ is the prescribed initial condition on $u$. We solve the above partial differential equation by discretizing via a DG finite element method that is based on Nitsche's method (Nitsche [1970]) to weakly enforce Dirichlet boundary conditions. In our DG framework this method enables us to weakly enforce the continuity in $u$ across the non-conforming interface.

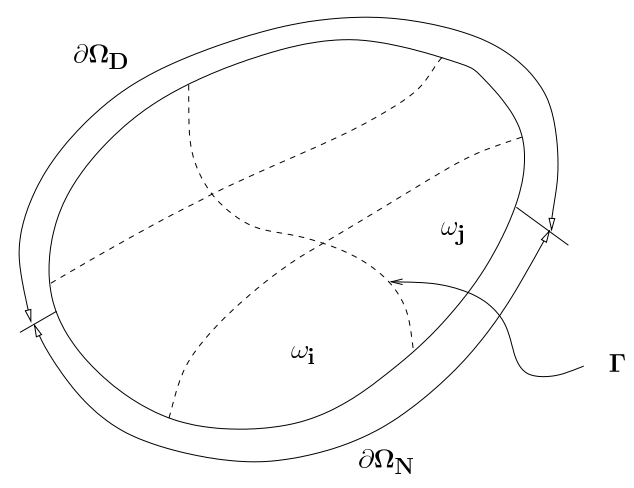

Fig. 1. Domain Partitioning

The region $\Omega$ is divided into $n$ non-overlapping sub-domains $\omega_{1}, \ldots, \omega_{n}$ with boundaries $\partial \omega_{1}, \ldots, \partial \omega_{n}$ such that $\Omega=\cup_{i=1}^{n} \omega_{i}$. Denoting the set of all interior boundaries as $\Gamma$, we have:

$$
\Gamma=\cup e_{i j}
$$


where $e_{i j}=\overline{\partial \omega_{i}} \cap \overline{\partial \omega_{j}}$ is the interior boundary shared by $\omega_{i}$ and $\omega_{j}$. On $e_{i j}$ we define the jump and average operators as

$$
\begin{aligned}
\llbracket u \rrbracket & =\left.u\right|_{\partial \omega_{i}}-\left.u\right|_{\partial \omega_{j}} \\
\langle\langle u\rangle\rangle & =\frac{1}{2}\left(\left.u\right|_{\partial \omega_{i}}+\left.u\right|_{\partial \omega_{j}}\right)
\end{aligned}
$$

where $i<j$. In what follows we describe our DG formulation and relate the final weak statement to the underlying partial differential equations. Standard Galerkin finite element formulations employ test and trial functions that are continuous in $\Omega$. With the DG formulation these functions are no longer continuous across $\Gamma$, rather they belong to the following spaces:

$$
V_{h}=\left\{w_{h} \in L_{2}(\Omega):\left.w_{h}\right|_{\omega_{i}} \in \mathbb{R}^{p}\left(\omega_{i}\right), \text { for } p \geq 1\right\}
$$

with $h$ being the maximal length of the sides of our quasi-uniform triangulation. Assuming sufficiently regular boundary and source data we require our DG formulation to weakly satisfy the following additional conditions on any interior interface and particularly on $\Gamma$, i.e.:

$$
\begin{aligned}
\llbracket u_{h} \rrbracket=0 & & \text { on } \Gamma \times I \\
\llbracket \nabla u_{h} \rrbracket \cdot \mathbf{n}_{i}=0 & & \text { on } \Gamma \times I
\end{aligned}
$$

Thus, to formulate the weak form of the partial differential equation, we weight equations (1), (3), (8), and (9) by $w_{h}, w_{h},-\left\langle\left\langle\nabla w_{h}\right\rangle\right\rangle \cdot \mathbf{n}$, and $\left\langle\left\langle w_{h}\right\rangle\right\rangle$ respectively, and integrate over their respective domains to obtain:

$$
\begin{aligned}
& \sum_{i} \int_{\omega_{i}} w_{h}\left(\dot{u}_{h}-\Delta u_{h}-f\right) d \Omega+\int_{\partial \Omega_{N}} w_{h}\left(\nabla u_{h} \cdot \mathbf{n}\right) d s \\
& \quad-\int_{\Gamma}\left(\left\langle\left\langle\nabla w_{h}\right\rangle\right\rangle \cdot \mathbf{n}\right) \llbracket u_{h} \rrbracket d s+\int_{\Gamma}\left\langle\left\langle w_{h}\right\rangle \rrbracket \nabla u_{h} \rrbracket \cdot \mathbf{n} d s=0\right.
\end{aligned}
$$

This weak form could lead to instabilities (Becker and Hansbo [1999]), so we stabilize our formulation by augmenting the above with the following penalty function:

$$
P=\int_{\Gamma} \frac{\eta}{h} \llbracket w_{h} \rrbracket \llbracket u_{h} \rrbracket d s
$$

which is related to the jump condition on $u^{h}$ (cf. equation (8)). Integration by parts, and the identity $\llbracket a b \rrbracket=\llbracket a \rrbracket\langle\langle b\rangle\rangle+\langle\langle a\rangle\rangle \llbracket b \rrbracket$ yields the DG problem statement:

Find $u_{h}: \Omega \times I \rightarrow \mathbb{R}$ where $u_{h}(t) \in V_{h}$ such that

$$
\begin{aligned}
\left(\dot{u}_{h}, w_{h}\right)+a\left(u_{h}, w_{h}\right) & =\left(f, w_{h}\right) \quad \forall\left(w_{h}, t\right) \in V_{h} \times I \\
\left(u_{h}(0)-\tilde{u}, w_{h}\right) & =0 \quad \forall w_{h} \in V_{h}
\end{aligned}
$$

where $\left(u_{h}, w_{h}\right)$ is the standard $L_{2}$ inner product over $\Omega$ and 


$$
\begin{aligned}
a\left(u_{h}, w_{h}\right)= & \sum_{i} \int_{\omega_{i}} \nabla w_{h} \cdot \nabla u_{h} d \Omega-\int_{\Gamma} \llbracket w_{h} \rrbracket\left\langle\left\langle\nabla u_{h}\right\rangle\right\rangle \cdot \mathbf{n} d s-\int_{\Gamma} \llbracket u_{h} \rrbracket\left\langle\left\langle\nabla w_{h}\right\rangle\right\rangle \cdot \mathbf{n} d s \\
& +\int_{\Gamma} \frac{\eta}{h} \llbracket w_{h} \rrbracket \llbracket u_{h} \rrbracket d s
\end{aligned}
$$

For the steady-state case, i.e. $\dot{u}=0$, our weak form is the same as that of Becker and Hansbo [1999], and Arnold et al. [2002], and hence their stability and optimal convergence proofs hold. Our formulation leads to a sparse, symmetric system of equations thereby maintaining the computational efficiency of the regular finite element approach. Though, Arnold [1982], Rivière and Wheeler [2000], have formulated two different non-symmetric weak forms for solving nonlinear parabolic equations, to our knowledge there is no literature where our proposed DG scheme has been used for parabolic equations.

\section{A priori analysis}

In this section we highlight the important results of our a priori analysis. One can refer to Kulkarni et al. [2005] for a detailed description of the a priori analysis. Through our a priori analysis we demonstrate that our methodology is consistent, stable and converges at a rate similar to that of a standard Galerkin scheme. The following lemma, an extension of that in Becker and Hansbo [1999] proves consistency i.e. the exact solution to the partial differential equation (1)-(4) satisfies the DG weak form (12).

Lemma 1. If $u$ is the solution to equations (1)-(4) then it also solves (12)

Proof. Since $u$ solves equations (1)-(4) $\llbracket u \rrbracket=0,\langle\langle u\rangle\rangle=u,\langle\langle\nabla u\rangle\rangle \cdot \mathbf{n}=\nabla u \cdot \mathbf{n}$. Thus

$$
\begin{aligned}
\left(f, w_{h}\right)-a\left(u, w_{h}\right) & =\left(f, w_{h}\right)-\left(\dot{u}, w_{h}\right)-\left(\nabla u, \nabla w_{h}\right)+\int_{\Gamma} \llbracket w_{h} \rrbracket \nabla u \cdot \mathbf{n} d s \\
& =\left(f-\dot{u}+\Delta u, w_{h}\right) \\
& =0
\end{aligned}
$$

which proves the lemma.

For our error analysis we introduce $\mathcal{H}^{r}$ the standard Hilbert space with its associated norm $\|\cdot\|_{\mathcal{H}^{r}(\Omega)}$. Since the DG formulation allows for a discontinuous field across $\Gamma$ we introduce the following norm that accounts for the discontinuity in $u_{h}$ :

$$
\left\|\left|u_{h}\right|\right\|^{2}=\left\|\nabla u_{h}\right\|_{L_{2}(\Omega)}^{2}+\left\|h^{1 / 2}\left\langle\left\langle\nabla u_{h}\right\rangle\right\rangle \cdot \mathbf{n}\right\|_{L_{2}(\Gamma)}^{2}+\left\|h^{-1 / 2} \llbracket u_{h} \rrbracket\right\|_{L_{2}(\Gamma)}^{2}
$$

This norm is equivalent to a $\mathcal{H}^{1}$ norm on a broken space. We now state without proof our a priori error estimate: 
Theorem 1. If $u_{h}$ is the solution of (12) and $u$ is the solution of (1)-(4), then

$$
\begin{aligned}
\left\|u_{h}-u\right\| \mid \leq & C \mid\left\|u_{h}(0)-\tilde{u}\right\| \| \\
& +C h^{r-1}\left(\|\tilde{u}\|_{\mathcal{H}^{r}(\Omega)}+\|u(t)\|_{\mathcal{H}^{r}(\Omega)}+C_{2}\left(\int_{0}^{t}\|\dot{u}\|_{\mathcal{H}^{r}(\Omega)}^{2} d z\right)^{1 / 2}\right) \\
& \text { for } \quad 2 \leq r \leq p+1 \quad u \in \mathcal{H}^{r}(\Omega) \cap \mathcal{H}_{0}^{1}(\Omega)
\end{aligned}
$$

The first term on the right side of the inequality accounts for the error in projecting the initial condition onto the finite element space; weak satisfaction of the initial condition via equation (13) would ensure that this term converges at an optimal rate of $h^{r-1}$. The second term shows that our error converges at the rate of $h^{r-1}$ which represents an optimal order of convergence in the $|\| \cdot \cdot|||$ norm.

The following lemma (also stated without proof), an extension of that in Johnson [1987] shows the stability of our DG formulation i.e. under suitable assumptions on the smoothness of the initial condition the DG solution remains bounded and decays over time.

Lemma 2 (Stability). Let $u_{h}$ be the solution to (12) with $f=0$ then it satisfies the property

$$
\left\|u_{h}(t)\right\|_{L_{2}(\Omega)} \leq\left\|u_{h}(0)\right\|_{L_{2}(\Omega)} \leq\|\tilde{u}\|_{L_{2}(\Omega)} \quad \forall t \in I
$$

This lemma which uses coercivity of the bilinear operator $a\left(u_{h}, w_{h}\right)$ proves the stability of the DG formulation for the case when $f=0$.

\section{Numerical Results}

To validate the DG formulation and the a priori analysis we consider equation (1) with initial condition $\tilde{u}=\sin (x)$ over a 1-D domain $\Omega=(0, \pi)$. The analytical solution for this problem is Kreyszig [1993]

$$
u(x, t)=e^{-t} \sin (x)
$$

The numerical examples employ linear elements and a backward-Euler time stepping scheme. Each element is considered to be a separate sub-domain $\omega_{i}$ and the interface $\Gamma$ is the collection of all the end points of the elements. In figure (2) we plot $u(x, t)$ at various instants in time. As expected $u$ decays with increasing time. Figures 3(a), 3(b) illustrate the error norm (cf. equation (16)) versus the element size $h$. Plot 3(a) which is obtained using a time step of $\Delta t=0.0001$, and figure 3(b) obtained by varying the time step as $\Delta t \propto h^{2}$ show the optimal order of error convergence.

To further validate the DG formulation we repeat the above example using the hat function initial condition

$$
\tilde{u}=\left\{\begin{array}{lll}
x, & \text { if } & 0<x \leq \pi / 2 \\
\pi-x & \text { if } & \pi / 2<x<\pi
\end{array}\right.
$$




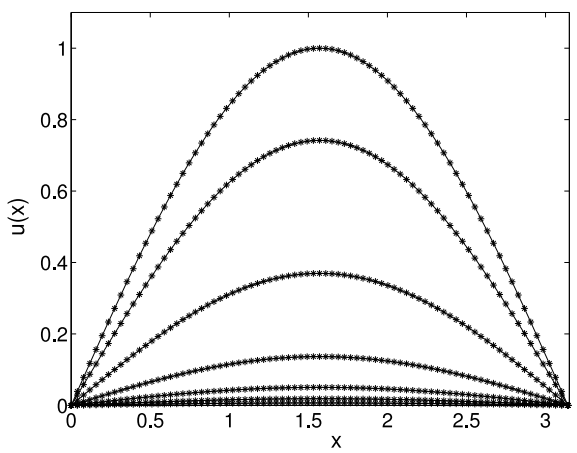

Fig. 2. $u(x)$ at various time steps

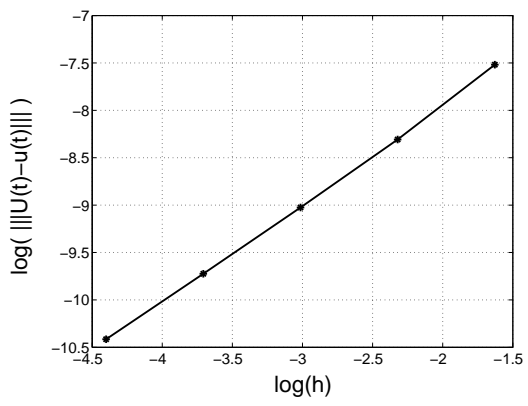

(a)

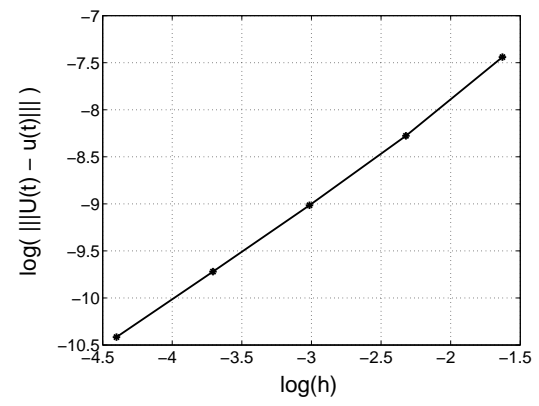

(b)

Fig. 3. Error norm with (a) $\Delta t=0.0001$ and (b) with $\Delta t \propto h^{2}$

for which the analytical solution is Kreyszig [1993]

$$
u(x, t)=\sum_{n=1}^{\infty} B_{n} \sin (n x) e^{-n^{2} t}
$$

where

$$
B_{n}=\left\{\begin{array}{rll}
\frac{4}{n^{2} \pi} & \text { for } & n=1,5,9, \ldots \\
-\frac{4}{n^{2} \pi} & \text { for } & n=3,7,11, \ldots
\end{array}\right.
$$

In figure 4(a) we plot the evolution of $u$ over time. Though the initial condition in this example problem is not as smooth as in the previous example figure 4(b) shows that we still obtain optimal convergence rate. 


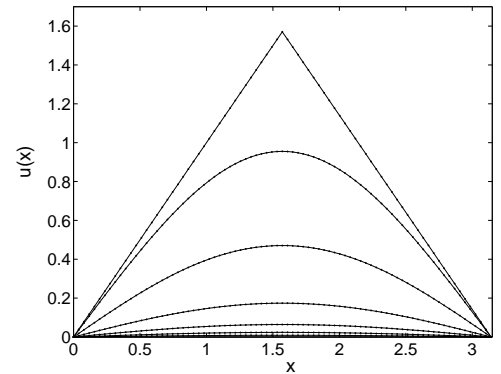

(a)

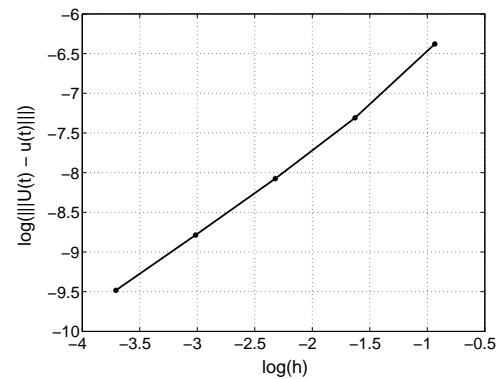

(b)

Fig. 4. Simulation with hat function as initial condition (a) $u(x)$ at various time steps (b) Error with $\Delta t=0.001$

\section{Conclusions}

A DG formulation for solving parabolic equations on non-conforming meshes has been developed. The formulation leads to a symmetric, sparse system and does not involve constraint equations or Lagrange multiplier fields like the mortar method. The a priori analysis of the method shows that the method is consistent, stable and demonstrates optimal order of convergence. Numerical results validate our analysis. We believe the method has applications to efficient multi-component simulation and adaptive refinement. Currently we are applying this scheme to adaptively refine interface evolution problems (Kulkarni et al. [2005]).

\section{References}

T. Arbogast. Non-mortar mixed finite element method for elliptic problems on nonmatching multiblock grids. Computer Methods in Applied Mechanics and Engineering, 149(1-4):255-265, 1997.

D. N. Arnold. An interior penalty finite element method with discontinuous elements. SIAM Journal of Numerical Analysis, 19:742-760, 1982.

D. N. Arnold, F. Brezzi, B. Cockburn, and L. D. Marini. Unified analysis of discontinuous galerkin methods for elliptic problems. SIAM Journal of Numerical Analysis, 39:1749-1779, 2002.

R. Becker and P. Hansbo. A finite element method for domain decomposition with non-matching grids. INRIA Tech. Report 3613, 1999.

C. Bernadi, Y. Maday, and A. Patera. A new nonconforming approach to domain decomposition: the mortar element method. In H. Brezis and J. L. Lions, editors, Nonlinear partial differential equations and their application. Pitman, 1989. 
E.G.D. Carmo and A.V.C. Duarte. A discontinuous finite-element based domain decomposition method. Comp. meth. in applied mech. and engrg., 190:825-843, 2000.

B. Cockburn, G.E. Karniadakis, and C.-W.Shu. Discontinuous Galerkin methods. Lecture notes in computational science and engineering. Springer Verlag, 11 edition, 2000.

L. Demkowicz, J.T. Oden, W. Rachowicz, and O. Hardy. Toward a universal h$\mathrm{p}$ adaptive finite element strategy, Part 1 . Constrained approximation and data structure. Computer Methods in Applied Mechanics and Engineering, 77:79-112, 1989.

B. Guo and I. Babuška. The h-p version of the finite element method, parts 1 and 2. Computational Mechanics, 1:21-41, 203-220, 1986.

Claes Johnson. Numerical solution of partial differential equations by the finite element method. Cambridge university press, 2nd edition, 1987.

E. Kreyszig. Advanced engineering mathematics. Wiley eastern, $5^{\text {th }}$ edition, 1993.

D. V. Kulkarni, D. V. Rovas, and D. A. Tortorelli. Discontinuous galerkin framework for adaptive solution of interface evolution problems. To be submitted to Intern. Journal Numer. Meth. Engrg., 2005.

J. Nitsche. Über ein variationsprinzip zur lösung von Dirichlet-problemen bei verwendung von teilräumen, die keinen randbedingungen unterworfen sind. $A b h$. Math. Univ. Hamburg, 36:9-15, 1970.

I. Perugia and D. Schötzau. On the coupling of local discontinuous Galerkin and conforming finite element methods. J. Sci. Comput., 16(4):411-433, 2001.

B. Rivière and M. F. Wheeler. A discontinuous Galerkin method applied to nonlinear parabolic equations. In B. Cockburn, G. E. Karniadakis, and C.-W. Shu, editors, Discontinuous Galerkin methods. Theory, Computation and applications, volume 11 of Lecture Notes in Computational Science and Engineering, pages 231-244. Springer Verlag, February 2000.

B . I. Wohlmuth. A comparison of dual Lagrange multiplier spaces for mortar finite element discretizations. Math. Modelling and Numer. Analysis, 36(6):995-1012, 2002. 